\title{
The level of anxiety in women with graves' disease
}

\section{Relevance}

Thyroid gland (TG) diseases have a leading place among all forms of endocrine pathology. One of the most common distyroid conditions is Graves' disease (GD), which occurs with the frequency of 23 cases per 100 thousand populations, and recently this indicator has increased. ${ }^{1-4}$ Different changes in thyroid gland function during GD lead to numerous clinical manifestations because of the nervous system damage. Over the past decades only scattered information about the specific neurological syndromes caused by thyrotoxicosis has been presented in the literature, which does not show the diversity of pathology forms and their pathomorphosis in connection with the modern type and severity of the goiter. Meanwhile, because GD often damages the nervous system, doctors in the late XIX-early XX century called that condition "neurotireosis" and "thyreoneurosis," thereby emphasizing the mandatory involvement in the pathological process of the central nervous system. ${ }^{5-11}$ It is known that a fairly frequent symptom of hyperthyroidism is muscle tremor, a decrease of muscle strength, a change in tendon reflexes, and other disorders of the nervous system. ${ }^{2-12}$ Sometimes in this condition, neuromuscular disorders come to the fore, significantly overtaking other manifestations of the disease. At the same time, with the normalization of thyroid function, electroneuromyographic changes disappear much later than vegetative and somatic signs of thyrotoxicosis, which may serve as a criterion for the complete clinical recovery. Typically in this condition complaints include increased excitability, irritability, fussiness, tearfulness, excessive motor activity, emotional instability with a rapid change of mood from agitation to depression. During severe thyrotoxicosis it is not uncommon when mental disorders reach the severity of a psychotic or depressive disorder, but apathetic-abulic syndrome can be noted rarely. There are possibilities of other changes, such as changes in the level of consciousness (psychomotor agitation, sopor), up to dementia. Thus, the pathology of the neuromuscular system includes myasthenic syndrome in $10-75 \%$ of patients, encephalopathy in $70.8-91.5 \%$ and polyneuropathy in $0-4.7 \% .^{2-3}$ Based on the abovesaid, we set the goal: to study the level of anxiety in women with GD depending on the activity of the disease.

\section{Materials and methods}

81 women with GD were examined during inpatient treatment in the department of "Thyroid pathology" and at consultative polyclinic of the Republican Specialized Scientific and Practical Medical Center of Endocrinology (RSSPMCE).The average age of the patients was 33.7 7.4 years. According to the functional state of the thyroid gland, women are divided into the following groups: 1-group, patients with GD in the stage of thyrotoxicosis, $n=45$ women (55.6\%). 2-group, patients with GD in the stage of remission of thyrotoxicosis, $n=36(44.4 \%)$. The control group consisted of 28 healthy women of the same age (mean age $30.5 \pm 5.3$ years) without thyroid dysfunction. The average duration of the disease in all patients was $3.5 \pm 0.8$ years. Table 1 Women in-group 1 received thyreostatics (mercazolil or tyrosol) in an individually selected dose. In addition to thyreostatics, detoxification, antianemic and, if necessary symptomatic treatment were prescribed. The average dose of thyreostatics was 20-30 mg per day. Treatment with thyreostatics was carried out under the control of free thyroid hormones and TSH levels in blood serum. Group 2 was
Special Issue - 2018

\author{
Ubaidullaeva NB, Allayarova GI, \\ Nasyrkhodzhaev Ya B, Ismailov SI, Juraeva \\ $\mathrm{NA}$, Almuradov FF
}

Tashkent Pediatric Medical Institute, Uzbekistan

Correspondence: Ubaydullaeva Nilufar Bahromhodjaevna, Republican Specialized Scientific and Practical Medical Center of Endocrinology named after academician Yo.Kh.Turakulov, Tashkent Pediatric Medical Institute, Uzbekistan, Email nilufarhan@inbox.ru

Received: August 14, 2018 | Published: November 15, 2018

consisting of women who received conservative treatment for 12-18 months, during this time, the levels of TSH and free T4 were in the normal ranges. After consultation with the endocrinologist and before the beginning of thyreostatic treatment, patients were consulted by a neurologist on the basis of the RSSPMCE. For more detailed study of the neuropsychological status and cognitive impairment SpielbergerKhanin test has been used. Testing using the Spielberger-Khanin method is carried out using two forms: one for measuring indicators of situational anxiety (reactive anxiety, statements No.1-20), and the second for measuring the level of personal anxiety (personal anxiety, statements No. 21-40). In all patients, we have assessed levels of TSH, free T4 (anti-T4), antibodies to TPO in serum by the RIA method using commercial sets of the firm "Immunotech" (Czech Republic) on the basis of RSSPMCE. The regulatory levels of hormones by this method were as following: fT4-11.5-23.0pmol/ L; TSH-0.174.05mME/L, antibodies to TPO above $12 \mathrm{IU} / \mathrm{ml}$. All patients underwent an ultrasound examination of the thyroid gland with the determination of its volume using an ultrasound scanner "USSIM-5000 FUCUDA-U2000 (Japan)" equipped with a linear sensor 7.0MHz. The results of the research were inserted into the Microsoft Excel program. Statistical analysis of the results of the study was arried out using the statistical program STATISTICA 6 and Biostat.

Table I Spreading of women by groups

\begin{tabular}{lll}
\hline Groups & Number of women & Average age \\
\hline $\begin{array}{l}\text { I-group of GD in the stage of } \\
\text { thyrotoxicosis }\end{array}$ & $\mathrm{n}=45$ & $33.5 \pm 6.4$ years \\
$\begin{array}{l}\text { 2-group GD in the stage of } \\
\text { remission of thyrotoxicosis }\end{array}$ & $\mathrm{n}=36$ & \\
$\begin{array}{l}\text { Control group, healthy } \\
\text { women }\end{array}$ & $\mathrm{n}=28$ & $30.5 \pm 5.3$ years \\
\end{tabular}

\section{Results}

The average duration of the disease in all patients was $3.8 \pm 1,4$ years. Estimation of thyroid status by the results of thyroid hormone levels and serum TSH in the patients of the 1 st group revealed that the level of fT4 was elevated and the level of TSH was decreased, which corresponded to the thyrotoxicosis. The indicators of fT4 and $\mathrm{TSH}$ of blood serum in the 2 nd group were within the normal ranges (Figure 1). According to the goal, we evaluated neurological and neuropsychological status, depending on the activity of the disease. 
The Spielberger-Khanin test showed a high level of reactive anxiety (RA) is seen in $17(54.8 \%)$ patients with thyrotoxicosis, and a high level of personal anxiety (PA) for $18(58.1 \%)$ patients. In women with GD in the stage of thyrotoxicosis remission, we have seen a low level of RA (14-46.7\%) and PA (14-46.7\%). In the control group, there was only a low level of RA (4-14.3\%) and PA (3-10.7\%) (Figure 2). Integral assessment of RA of patients with GD in the stage of thyrotoxicosis was $36.9 \pm 11.6$ points, PA $39.3 \pm 13.7$ points. In the group with GD in the stage of remission, thyrotoxicosis RA and PA in total $29.5 \pm 12.8$ points and $27.4 \pm 14$.1points, respectively. In the control group, RA and PA parameters were in total $5.3 \pm 3.3$ points and $4.3 \pm 1.3$ points, respectively. The reactive anxiety in patients with GD in the stage of thyrotoxicosis was manifested by a feeling of stiffness and inability to relax (in 48.4\%), anxiety (in 51.6\%), tension (38.7\%), feelings of unrest (19.4\%), a sense of anxiety due to palpitation (in $12.9 \%$ ), attention disorders (in $6.5 \%$ ).Personal anxiety manifested with tearfulness (in $48.4 \%$ ), with a feeling of fatigue $(51,6 \%)$ and doubt in the succeeding of treatment (in 22.6\%). In patients with GD in the stage of remission, thyrotoxicosis RA was manifested by anxiety (10.0\%), a sense of unrest (in 10.0\%), a sense of anxiety due to palpitations (in 13.3\%).Personal anxiety manifested with tearfulness $(6.7 \%)$ and weakness $(6.7 \%)$.

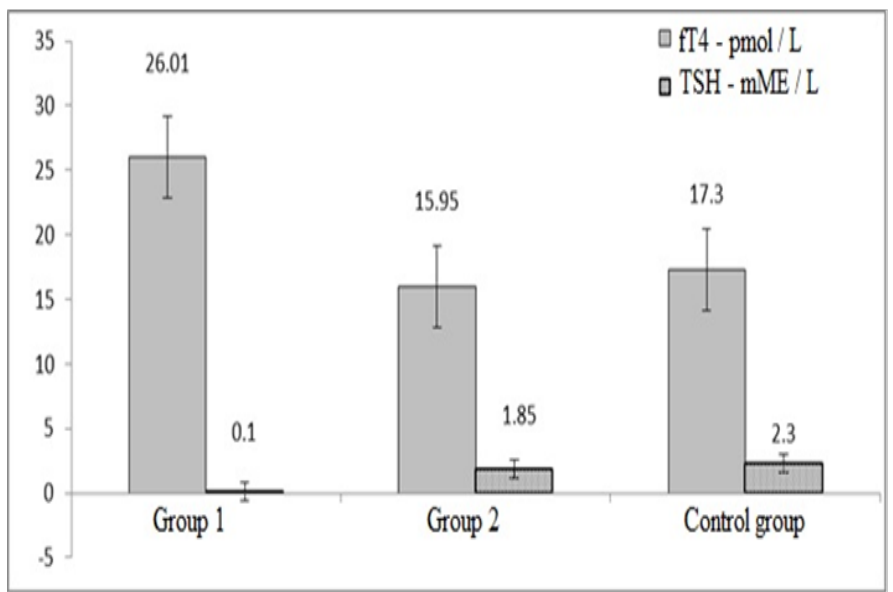

Figure I The indicators of TSH and fT4 in women with GD.

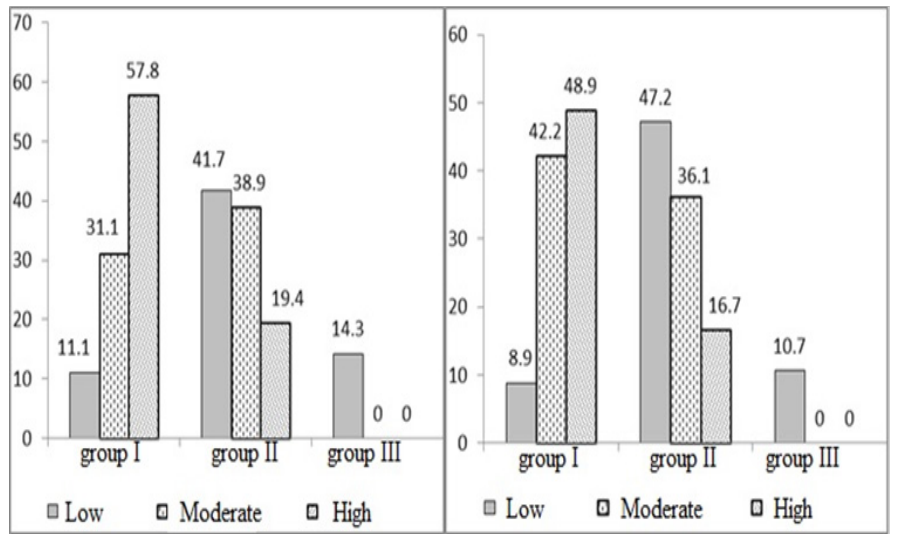

Figure 2 Indices of levels of reactive and personal anxiety by the SpielbergerKhanin scale.

\section{Discussion}

The obtained data shows an undoubted unfavorable effect of excess thyroid hormones on the CNS state, which is confirmed by the literature data. ${ }^{7-12}$ Moreover, we managed to establish that even in the absence of active complaints from the patient as a result of a detailed neuropsychological examination; certain pathological changes had been detected. Our study showed that the peculiarity of the neuropsychological status in patients with GD is a high level of anxiety (both personal and situational), absent-mindedness, short temper, attention disturbance, sleep disturbance, while memory indicators remain intact. Thus, it can be concluded that thyrotoxicosis is involved in the nervous system activity, which indicates the need for a detailed neurological examination of this category of patients with the purpose of early detection of the nervous system disturbances and to prescribe appropriate therapy. According to literary data, in rare cases, there is a possibility of delirium with hyperkinesia, in elderly patients - apathy, depression, drowsiness. Mental disorders occurred not only with high, but also with a moderate increase of thyroid hormones in blood serum, and, apparently due to not only thyrotoxicosis, but also because of metabolic disorders.

\section{Conclusion}

As a result of the analysis of the obtained data, we can formulate followings:

i. An increase in the level of thyroid hormones leads to a negative effect on the structure of the central nervous system.

ii. Pathological changes in the part of neuropsychological and neurological status are revealed even in patients who do not make active complaints to the neurologist.

iii. The Spielberger-Khanin test showed a high level of RA is seen in $54.8 \%$ patients with thyrotoxicosis, and a high level of personal anxiety PA for $58.1 \%$ patients. In women with GD in the stage of thyrotoxicosis remission, we have seen a low level of RA $46.7 \%$ and PA $46.7 \%$.

iv. Psychoemotional disorders and autonomic disorders prevailed in patients with GD in the stage of thyrotoxicosis, while in the structure of psychoemotional disorders, neurotic-like conditions, confirmed by the results of neuropsychological testing, appeared to the fore.

\section{Acknowledgments}

None.

\section{Conflict of interest}

The author declares no conflict of interest.

\section{References}

1. Abraham NM, Wallin G, Trdisk F, et al. Thyroid associated opthalmopathy; quality of life, follow-up of patients, randomized to treatment with antithyroid drugs or radioiodine. Eur J Endocrinol. 2010;163(4):651-657.

2. Bensenor IM, Lotufo PA, Menezes PR, et al. Subclinical hyperthyroidism and dementia: the Sao Paulo A geing \& Health Study (SPAH). BMC Public Health. 2010;10:298-302.

3. Zaitsev IA, Kirienko VT, Zhidkikh VN, et al. Evaluation of the quality of life in healthy individuals. Visnik morskoi meditsinini. 2010;2(48):30-36.

4. Ismailov SI, Ubaydullaeva NB, Mirzaeva UZ. The guality of women with Graves disease after radioiodine therapy. European Science Review. 2016:49-54.

5. Ismailov SI, Akbutayev AM, Elov AA. Quality of life of patients on the background of therapy with thyroxine and a combination of thyroxine and triiodothyronine after total thyroidectomy due to Graves' disease. 
International Endocrinology Journal. 2014;5(61):52-55.

6. Ismailov SI, Ubaidullaeva NB, Nasyrkhodzhaev Ya B, et al. Pregnancy and outcomes of labor in women with diffuse toxic goiter after radioiodine therapy. Infection, immunity, and pharmacology. 2014;5:66-72.

7. Kim JM, Stewart R, Kim SY, et al. Thyroid stimulating hormone, cognitiveimpairment and depression in an older Korean population. Psychiatry Investig. 2010;7(4):264-269.

8. Cramon P, Winther K, Watt T, et al. Quality-of-Life Impairments Persist Six Months After Treatment of Graves' Hyperthyroidism and Toxic Nodular Goiter. A Prospective Cohort Study Thyroid. 2016;26(8):1010-1018.

9. Placidia GPA, Boldrinia M, Patronellia A, et al. Prevalence of
Psychiatric Disorders in Thyroid Diseased Patients. Neuropsychobiology. 1998;38(4):222-225.

10. Ritchie M, Yeap BB. Thyroid hormone: Influences on mood and cognition in adults. Maturitas. 2015;81(2):266-275.

11. Vadiveloo T, Donnan PT, Cochrane L, et al.The Thyroid Epidemiology, Audit, and Research Study (TEARS): morbidity in patients with endogenous subclinical hyperthyroidism. J Clin Endocrinol Metab. 2011;96(5):1344-1351.

12. Shah MS, Davies TF, Stagnaro Green A. The thyroid during pregnancy: a physiological and pathological stress test. Minerva Endocrinol. 2003;28(3):233-245. 